\title{
La quimioterapia paliativa no mejoraría la calidad de vida de pacientes con cáncer en fase terminal
}

Palliative chemotherapy in patients with end-stage cancer would not improve quality of life

\section{Objetivos}

Evaluar la asociación entre el uso de quimioterapia y la calidad de vida cerca de la muerte, en relación con el estado funcional de los pacientes.

\section{Diseño y lugar}

Estudio de casos y controles anidado en una cohorte longitudinal multicéntrica, realizada en seis centros especializados en oncología de EE.UU.

\section{Pacientes}

Participaron 661 adultos reclutados entre 2002 y 2008 con diagnóstico de cáncer en fase terminal (metástasis a distancia o enfermedad refractaria a una o más líneas de quimioterapia) y expectativa de vida menor a seis meses. Debían contar con un cuidador formal y hallarse en capacidad para realizar una entrevista.

\section{Evaluación de factores pronósticos}

Cada individuo fue entrevistado una vez a una mediana de 3,8
Prigerson H. JAMA Oncol. 2015 Sep;1(6):778-84. meses antes de la muerte. La información sobre las enfermedades se obtuvo de las historias clínicas, y el número y la gravedad de las comorbilidades se evaluó de acuerdo al índice de Charlson. El estado funcional se evaluó a través de la escala del ECOG (Eastern Cooperative Oncology Group), en la que un mayor puntaje implica un peor estado funcional). Todos los pacientes fueron seguidos hasta su muerte. La calidad de vida cerca de la muerte se determinó utilizando encuestas a cuidadores, que calificaron el malestar físico y mental de los pacientes durante su última semana de vida.

\section{Medición de resultados principales}

A una media de 2,4 semanas después de la muerte del paciente índice se le pidió al cuidador que más lo había asistido, que puntuara en una escala de 0 a 10 varios aspectos: angustia psicológica, sufrimiento físico y calidad de vida en general .

\section{Resultados Principales}

Se resumen en la tabla 1.

Tabla 1. probabilidad de mejora en la calidad de vida durante la última semana de vida en usuarios de quimioterapia según el estado funcional basal de los pacientes

\begin{tabular}{|c|c|c|c|c|c|}
\hline \multirow{2}{*}{$\begin{array}{l}\text { Estado funcional } \\
\text { basal }\end{array}$} & \multirow{2}{*}{$\begin{array}{c}\text { Calidad de vida durante la } \\
\text { última semana }\end{array}$} & \multicolumn{2}{|c|}{ Uso de quimioterapia n (\%) } & \multirow{2}{*}{$\underset{\text { (IC95\%) }}{\text { OR }}$} & \multirow{2}{*}{$\mathbf{P}$} \\
\hline & & Si & No & & \\
\hline \multirow{2}{*}{$\begin{array}{l}\text { Bueno } \\
(n=122)\end{array}$} & Alta & $31(43,7)$ & $35(68,6)$ & \multirow{2}{*}{$\begin{array}{c}0,35 \\
(0,17 \mathrm{a} 0,75)\end{array}$} & \multirow[t]{2}{*}{0,01} \\
\hline & Baja & $40(56,3)$ & $16(31,4)$ & & \\
\hline \multirow{2}{*}{$\begin{array}{l}\text { Intermedio } \\
(\mathrm{n}=116)\end{array}$} & Alta & $27(49,1)$ & $29(47,5)$ & \multirow{2}{*}{$\begin{array}{c}1,06 \\
(0,51 \mathrm{a} 2,21)\end{array}$} & \multirow[t]{2}{*}{0,87} \\
\hline & Baja & $28(50,9)$ & $32(52,5)$ & & \\
\hline \multirow{2}{*}{$\begin{array}{l}\text { Malo } \\
(\mathrm{n}=58)\end{array}$} & Alta & $12(54,5)$ & $17(47,2)$ & \multirow{2}{*}{$\begin{array}{c}1,34 \\
(0,46 \text { a 3,89) }\end{array}$} & \multirow[t]{2}{*}{0,59} \\
\hline & Baja & $10(45,5)$ & $19(52,8)$ & & \\
\hline
\end{tabular}

\section{Conclusiones}

Aunque se utilizan esquemas de quimioterapia paliativa con el objeto de mejorar la calidad de vida de los pacientes con cáncer en fase terminal, su uso no mejoró la calidad de vida en aquellos con un estado funcional moderado o pobre, y la empeoró para los que mantenían buen estado general. La calidad de vida en el final de la vida en pacientes con cáncer terminal no mejora, y puede ser empeorada con el uso de quimioterapia, incluso en pacientes con buen estado general.

Fuente de financiamiento/conflicto de interés de los autores: National Institute of Mental Health, National Cancer Institute, National Institute of Minority Heath and Health Disparities, Weill Cornell Medical College y Department of Veterans Affairs. Algunos autores declararon haber asesorado a empresas farmacéuticas: Helsinn Therapeutics, Epi-Q, Boehringer Ingelheim, Pfizer, Otsuka, Estados Biosource Corporation, y EHE Intl.

\section{Comentario}

La decisión de ofrecer o no quimioterapia en pacientes al final de la vida es compleja, e implica múltiples dimensiones del vínculo entre médico y paciente (y grupo familiar). Para una mejor decisión resulta fundamental una adecuada (atenta, clara, empática y honesta) comunicación, y el respaldo de datos provistos por la investigación. Los autores reseñan adecuadamente la complejidad de esta cuestión ya planteada en la literatura ${ }^{1,2,3,4}$. Si bien existen publicaciones que documentaron las ventajas del uso de quimioterapia en pacientes con enfermedad oncológica avanzada, en general sólo comunicaron indicadores de sobrevida y no de la calidad de vida durante el estadio final de la misma, y menos aún en términos pragmáticos $5,6,7,8$

\section{Conclusiones del comentador}

Idealmente, la decisión de ofrecer quimioterapia paliativa a pacientes oncológicos cercanos al final de la vida debiera ser discutida entre el equipo tratante: médico de cabecera, oncólogo y equipo de cuidados paliativos. También resulta relevante la discusión sobre las motivaciones subjetivas y objetivas que motivan la indicación de estos esquemas de tratamiento en este grupo de pacientes.

Javier Vilosio [ Sección Cuidados Paliativos. Hospital Italiano de Buenos Aires. javier.vilosio@ hospitalitaliano.org.ar ]

Vilosio J. La quimioterapia paliativa no mejoraría la calidad de vida de pacientes con cáncer en fase terminal. Evid Act Pract Ambul 2016;19(4):118. Comentado de: Prigerson H y col. Chemotherapy Use, Performance Status, and Quality of Life at the End of Life. JAMA Oncol. 2015 Sep;1(6):77884. doi:10.1001/jamaoncol.2015.2378. PMID:26203912

\section{Referencias}

1. Meier D. 'I don't want Jenny to think I'm abandoning her': views on overtreatment. Health Aff (Millwood). 2014;33(5):895-898.

2. Bach P. The day I started lying to Ruth. 2014.http://nymag.com/news/features/cancer-peter-bach-2014-5/. Accedido el 17/4/2016.

3. Braga S. Why do our patients get chemotherapyuntil the end of life? Ann Oncol. 2011;22(11):2345-2348.

4. Anders $C y$ col. Treating in the dark:unanswered questions on costs and benefits of lateline therapy for metastatic breast cancer. Cancerlnvest. 2009;27(1):13-16.

5. Wagner A y col. Chemotherapy for advanced gastric cancer. Cochrane Database of Systematic Reviews 2010, Issue 3. Art. No.: CD004064. DOI: 10.1002/14651858.CD004064.pub3.

6. Albero A y col. Effectiveness of chemotherapy in advanced differentiated thyroid cancer: a systematic review.EndocrRelat Cancer. 2016 Feb;23(2):R71-84. doi: 10.1530/ERC-15-0194.

7. Best $L$ y col. Collaboration Colorectal Meta-analysis. Palliative chemotherapy for advanced or metastatic colorectal cancer. Cochrane Database of Systematic Reviews 2000, Issue 1. Art. No.: CD001545. DOI: 10.1002/14651858.CD001545.

8. Non-Small Cell Lung Cancer Collaborative Group. Chemotherapy and supportive care versus supportive care alone for advanced non-small cell lung cancer. Cochrane Database of Systematic Reviews 2010, Issue 5. Art. No.: CD007309. DOI: 10.1002/14651858.CD007309.pub2. 